\title{
Metal activated carbon as an efficient filler for high-density polyethylene nanocomposites
}

\author{
Muhammad Nisar $^{1} \quad$ | Pascal S. Thue ${ }^{2}$ | Myriam B. Maghous ${ }^{1}$ | Julian Geshev ${ }^{3}$ | \\ Eder C. Lima ${ }^{2}$ | Sandra Einloft ${ }^{1} \odot$
}

${ }^{1}$ Programa de Pós-Graduação em Engenharia e Tecnologia de Materiais (PGETEMA), School of Technology, Pontifical Catholic University of Rio Grande do Sul (PUCRS), Porto Alegre, Rio Grande do Sul, Brazil

${ }^{2}$ Graduate Program in Metallurgical, Mine and Materials Engineering (PPGE3M), School of Engineering, Federal University of Rio Grande do Sul (UFRGS), Porto Alegre, Rio Grande do Sul, Brazil

${ }^{3}$ Instituto de Física, Universidade Federal do Rio Grande do Sul, Porto Alegre, Rio Grande do Sul, Brazil

\section{Correspondence}

Sandra Einloft, Programa de PósGraduação em Engenharia e Tecnologia de Materiais (PGETEMA), School of Technology, Pontifical Catholic University of Rio Grande do Sul (PUCRS), Porto Alegre, RS, Brazil.

Email: einloft@pucrs.br

\section{Funding information}

Conselho Nacional de Desenvolvimento Científico e Tecnológico, Grant/Award Numbers: 305796/2016-0, 314429/2018-2, 422740/2018-7; Coordenação de Aperfeiçoamento de Pessoal de Nível Superior, Grant/Award Number: 001

\begin{abstract}
interesting properties of magnetic nanocomposites have attracted attention of both academic and industrial researchers. In this work, thermal, mechanical, morphological, and magnetic properties of polyethylene (PE) nanocomposites were inspected using carbon-based magnetic fillers ( $\mathrm{C}-\mathrm{Ni}, \mathrm{C}-\mathrm{Co}$, and $\mathrm{C}-\mathrm{Fe}$ ). The melt mixing method was employed to prepare the nanocomposites using small amounts of filler ranging up to $2 \mathrm{wt} \%$. Wood sawdust pyrolysis produces carbonized material activated by $\mathrm{Ni}, \mathrm{Co}$, or Fe salts and used as filler. The structural analysis was carried out using Fourier transform infrared spectroscopy indicating that the polymer chemical structure remains unaltered with the filler addition. Thermal stability of nanocomposites as well as the determination of metal amount in the carbon-based fillers was investigated by thermogravimetric analysis. Filler introduction enhanced the onset and the maximum degradation temperatures up to $11^{\circ} \mathrm{C}$ and $8^{\circ} \mathrm{C}$, respectively. The crystallization and melting temperatures examined by differential scanning calorimetry remained unchanged as compared to neat PE whereas the percent crystallinity was improved up to $8 \%$. The incorporation of the filler leads to the improvement in the elastic modulus of the polymer matrix. The addition of $2.0 \mathrm{wt} \%$ of the metal-carbonized filler in the diamagnetic polymer resulted in a thermoplastic nanocomposite with ferromagnetic behavior.
\end{abstract}

K E Y W O R D S

magnetic properties, metal activated carbon, nanocomposites, polyethylene

\section{1 | INTRODUCTION}

Polymer nanocomposites (PNCs) are combinations of polymer matrices with nanoscale materials, having exceptional physical, biological, and chemical properties. The PNCs have been extensively studied in the last two decades due to their wide applications in diverse areas. ${ }^{[1,2]}$ The introduction of nanofiller particles, such as clay nanosheets, graphite, and carbon nanotubes, enhances both the mechanical and thermal properties of the PNC as compared to the neat polymer matrix. ${ }^{[3-5]}$ Depending upon the nanofillers used, it can be transformed by creating thermal and/or electrical conductive network in the polymer matrix, ${ }^{[6,7]}$ hence giving rise to antimicrobial properties or improving fire-retardant characteristics. ${ }^{[8]} \mathrm{In}$ fact, the design of nanocomposites can aid in excessive applications ranging from high-strength structural materials to gas separation membranes. In recent years, synthesis of magnetic materials has attracted considerable attention due to their unique properties and applications 
in different fields such as magnetic recording, data storage, electronics, ferrofluids, bioprocessing, ${ }^{[9-11]}$ pharmaceutical areas, biomedical (ie, drug delivery) sensors, microwave absorption, and even environmental remediation. ${ }^{[12]}$ Consequently, magnetic PNCs have appealed inclusive attention for their varied potential applications such as energy storage devices, electrochromic devices, electronics, microwave absorbers, and sensors. ${ }^{[13-15]}$ The introduction of conventional magnetic nanoparticles such as nickel, iron, and cobalt leads to the magnetic PNCs. Besides the agglomeration problem associated with magnetic nanoparticles when used as filler in the polymer medium, easy oxidation is also an important issue to address. ${ }^{[16]}$ To overcome the challenges in dispersing the magnetic NPs, limited by the magnetically induced agglomeration and prevent them from oxidation, the chemical stabilization by encapsulating the magnetic core with carbon, polymer, silica, and surfactant has been reported. ${ }^{[17-21]}$

The excellent intrinsic properties of polyethylene (PE) such as high stiffness, improved tensile strength, low density, and its inertness toward several solvents and low cost made it one of the most important commercial plastics. Although to widen its industrial application, the physicochemical properties have to be further enhanced. ${ }^{[2]}$ Various techniques have been used to prepare polyolefin nanocomposites such as melt blending, solution mixing, and in situ polymerization. ${ }^{[4,5,23]}$ However, each of these methods presents some technical limitations, being solvent mixing method the less desirable from an economical and environmental point of view. In fact, based on PE and polypropylene (PP) nanocomposites the solvent mixing method is not practical meanwhile these polyolefins are generally used to solubilized in the solvents like trichlorobenzene and xylene at elevated temperature (eg, $120^{\circ} \mathrm{C}$ ), causing serious health problems. ${ }^{[24]}$ The in situ polymerization is considered to have uniform dispersion of the polymer matrix during the syntheses of the polymer from the monomer. However, the method has limited applicability and scalability. ${ }^{[25,26]}$ Melt blending is considered one of the supreme desired techniques owing to its industrial applicability, easy operation procedure, high yield, fast production rate, and solvent less process to avoid any health risk. ${ }^{[27]}$ More recently, we investigated the effect of different synthetic talc on the properties of the polyurethane nanocomposites. $^{[28-30]}$

The focus of the present work is to compare the use of different metal-activated carbons obtained from biomass ${ }^{[31]}$ (low cost source) as fillers to synthesize PE nanocomposites with good thermal, mechanical, and magnetic properties by the melt mixing technique.

\section{$2 \mid$ EXPERIMENTAL}

\subsection{Materials}

Commercial high-density PE (HDPE) (SGM9450F Braskem, Brazil) with $9.3 \mathrm{~g} / 10$ minutes $\left(21.6 \mathrm{~kg} / 190^{\circ} \mathrm{C}\right)$ melt flow rate, (Norm ASTM D-1238) having melting point of $125^{\circ} \mathrm{C}$ was introduced as polymer matrix. Small amount of antioxidant agent Irganox 1010 was also used during the composite synthesis.

The metal-carbonized materials containing different metals $(\mathrm{Fe}, \mathrm{Co}, \mathrm{Ni})$ were prepared by the reported method. ${ }^{[31]}$ Nanocomposites were prepared using Thermo Haake Rheomix-600 (Thermo Elec-tron Corp, Karlsruhe, Germany), working at $60 \mathrm{rpm}$ and $160^{\circ} \mathrm{C}$. The total amount of the filler, polymer, and small amount of Irganox1010 ( $\sim .005 \mathrm{~g})$ reach to $60 \mathrm{~g}$ per mixing. The different concentrations of the filler from 0 to $2 \mathrm{wt} \%$ were used. Initially, half of the HDPE ( $\sim 30 \mathrm{~g})$ and the antioxidant were melt blend. Subsequently, predefine percentage of filler was slowly introduced to the molten polymer after 2 minutes and the speed of the rotor was kept constant for more 3 minutes. Finally, the remaining approximately $30 \mathrm{~g}$ of the polymer pellets were introduced and mixed for more 5 minutes at a constant speed of $60 \mathrm{rpm}$. The total time of mixing was approximately 10 minutes. The heat pressed sheets were prepared at $160^{\circ} \mathrm{C}$ under the pressure of 200 bar.

\section{2 | Synthesis of activated carbons containing iron, cobalt, and nickel}

Ayous powder residue and metals $\left(\mathrm{Fe}^{2+}, \mathrm{Co}^{2+}\right.$, and $\left.\mathrm{Ni}^{2+}\right)$ chloride were used in the preparation of carbons composite materials. Ayous sawdust is a residue from sawmill industries. This biomass was kindly provided by sawmill industries from Ngaoundere Region, in Cameroon. It contains approximately $98 \%$ cellulose, hemicellulose, and lignin, with the remaining $2 \%$ made up of extractables (tannins, pectins, polymers with low degree of polymerization) and minerals. ${ }^{[32]}$

For the preparation of metal-activated carbons, a known amount of iron(II), cobalt(II), or nickel(II) chloride was dissolved in $80.0 \mathrm{~mL}$ of distilled water and $60.0 \mathrm{~g}$ of previously dried lignocellulosic biomass (with diameter $<250 \mu \mathrm{m}$ ) was added to the solution to form a paste. The proportion of the metal salt and the biomass was 1:1 (wt/wt). Afterward, the paste containing metals was dried overnight at $90^{\circ} \mathrm{C}$ to remove the water and then pyrolyzed in a quartz reactor as described in our previous study ${ }^{[31]}$ under a nitrogen gas atmosphere, with a flow rate of $200 \mathrm{~mL} \mathrm{~min}^{-1}$. The reactor was introduced in a 
conventional furnace and heated up for 60 minutes at $600^{\circ} \mathrm{C}$. Finally, the system was cooled down and the obtained carbon material was washed thoroughly, dried overnight at $105^{\circ} \mathrm{C}$, and stored for the synthesis of PNCs. For comparison with the nanocomposites, activated carbon (C-neat) was prepared under the same conditions but with no addition of metals.

\section{3 | CHARACTERIZATION}

The elemental analysis of Ayous powder and activated carbons filler was performed using an elemental analyzer (PerkinElmer M CHNS/O model 2400). A small amount $(0.05 \mathrm{~g})$, ovendried was used to determine total carbon containing $(\mathrm{C})$, nitrogen containing $(\mathrm{N})$, and hydrogen containing $(\mathrm{H})$. The oxygen-containing $(\mathrm{O})$ mass fraction was found by subtracting the ash, $\mathrm{C}, \mathrm{N}$, and $\mathrm{H}$ mass fractions from the total mass of the sample.

Fourier transform infrared (FTIR) spectra were recorded on a PerkinElmer Spectrum 100 spectrometer using attenuated total reflection mode in the range of 4000 to $400 \mathrm{~cm}^{-1}$. Transmission electron microscopy (TEM, Tecnia G2T20 FEI) was used to perform morphological analysis in order to investigate the degree of dispersion on the nanocomposites. Polymeric nanocomposites samples were prepared using an ultramicrotome RMC CXL with a thickness of approximately $50 \mathrm{~nm}$ at $-70^{\circ} \mathrm{C}$. The samples morphology was determined with a field emission scanning electron microscope (SEM) using Inspect F50 equipment (FEI Instruments) in secondary electrons mode. To avoid the charge accumulation, the deposited polymer on an aluminum stub was coated.

The magnetic characterization of the composite materials was accomplished by means of a vibrating sample magnetometer, model EZ9 MicroSense, with magnetic field $H$ ranging from -20 to $+20 \mathrm{kOe}$, at room temperature.

The melting and crystallization temperature as well as percent crystallinity were investigated by differential scanning calorimetry (DSC) (model DSC Q20) functional from $25^{\circ} \mathrm{C}$ to $180^{\circ} \mathrm{C}$ and heat rate of $10^{\circ} \mathrm{C} \mathrm{min}^{-1}$. The melting temperature $\left(T_{\mathrm{m}}\right)$ was determined in the second scan, and the relative percent crystallinity was intended as ratio of the melting enthalpy of the experimental sample to the completely crystalized PE melting enthalpy $\left(293 \mathrm{~J} \mathrm{~g}^{-1}\right)$. Thermal degradation and percent residue of the nanocomposites were analyzed by thermogravimetric analysis (TGA) using a SDT Q600 thermal analyzer Q20 (TA Instruments), samples were analyzed from $25^{\circ} \mathrm{C}$ to $800^{\circ} \mathrm{C}$ at a constant scanning rate of $20^{\circ} \mathrm{C} \mathrm{min}^{-1}$ using a constant flow of nitrogen $\left(100 \mathrm{~mL} \mathrm{~min}^{-1}\right)$ for polymeric nanocomposites and air for the carbon materials.

The stress-strain curve was measured according to ASTMD 638-10 at approximately $25^{\circ} \mathrm{C}$ using an $\mathrm{HP}$ model D-500 dynamometer. The results give the average value of five measurements tested for each wt $\%$ of the nanocomposite (typical deviation $~ 5 \%$ ) at a cross-head speed of $50 \mathrm{~mm} \mathrm{~min}^{-1}$.

A goniometer (Phoenix 300, SEO) was used to measure the static water contact angle (WCA) by the sessile drop method. The pictures were taken using "drop shape analysis system." Five drops of deionized water were applied to each sample and the mean of the angles was calculated, considering an experimental error of $2^{\circ}$ among the measurements.

\section{4 | RESULTS AND DISCUSSION}

\section{1 | Elemental analysis of the biomass and metal activated carbon}

Table 1 demonstrates the results of the elemental composition of activated carbons containing metal (nickel, cobalt, and iron). Compared to the precursor material, all the carbon-metal (C-M) samples exhibited high carbon and low oxygen contents. First, the biomass contained $6.26 \%$ hydrogen, $46.05 \%$ carbon, and $45.78 \%$ oxygen; however, once the pyrolysis and the washing process was carried out, the carbon contents in the $\mathrm{C}-\mathrm{Fe}, \mathrm{C}-\mathrm{Co}$, and C-Ni samples increased to $48.84 \%, 53.57 \%$, and $58.05 \%$,

\begin{tabular}{lllllll} 
Samples & \%C & $\mathbf{\% H}$ & $\mathbf{\% N}$ & $\mathbf{\% O}^{\mathbf{a}}$ & \%Ash $^{\mathbf{b}}$ & M-content (\%) \\
\hline Biomass & 46.05 & 6.26 & 0.33 & 45.78 & 1.56 & 0.00 \\
$\mathrm{Ni}-\mathrm{C}$ & 58.05 & 2.31 & 0.15 & 11.29 & 28.20 & 26.64 \\
$\mathrm{Co}-\mathrm{C}$ & 53.57 & 2.49 & 0.38 & 19.97 & 23.60 & 22.04 \\
$\mathrm{Fe}-\mathrm{C}$ & 48.84 & 1.54 & 0.10 & 1.72 & 47.80 & 46.24 \\
\hline
\end{tabular}

T A B L E 1 Elemental composition of biomass (C-neat) and activated carbons containing $\mathrm{Ni}, \mathrm{Co}$, and $\mathrm{Fe}$ 
respectively. This increase value of carbon content after carbonization in the presence of metal chlorides indicates that the aromatic structure dominates. ${ }^{[32]}$

It was also promising, using the TGA residue (or ash), to quantify the amount of iron, cobalt, or nickel present in the metal-carbon (M-C) samples after preparation. For instant, using the synthetic air atmosphere the ash content left represents the inorganic compound after the degradation of the sample. Determining the ash concentration of the biomass and $\mathrm{M}-\mathrm{C}$ samples, it is conceivable to find the metal concentration in the carbon composite (C-M) by subtracting. As seen in Table 1, the $\mathrm{C}-\mathrm{Fe}$ composite is the sample that showed more metal embedded in the structure of the carbon material (almost the same amount of metal as carbon), followed by $\mathrm{C}-\mathrm{Ni}$ and $\mathrm{C}-\mathrm{Co}$.

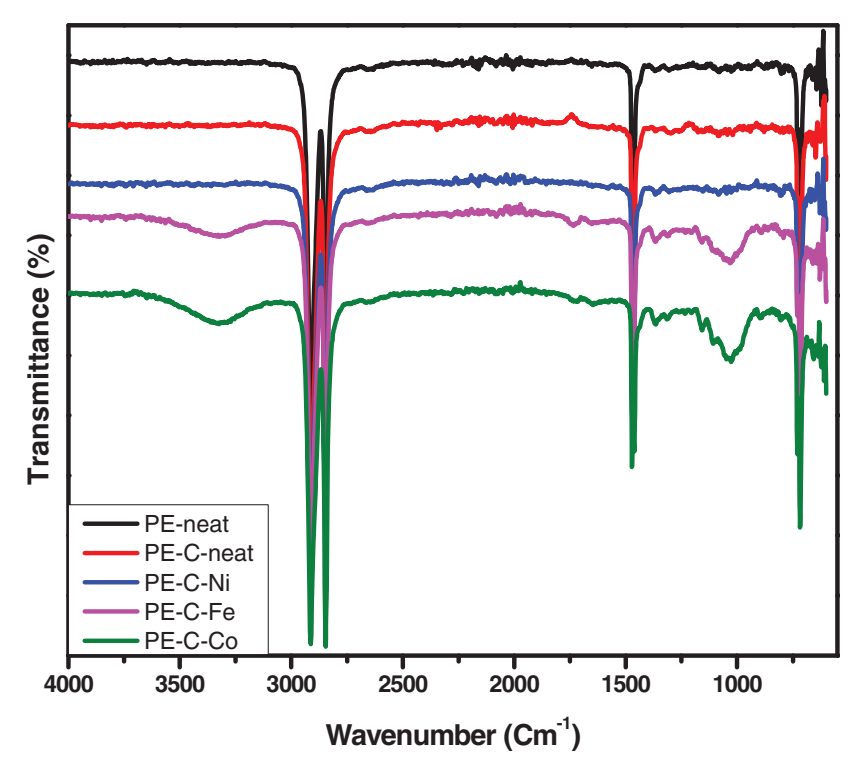

FI G URE 1 FTIR spectra of neat-HDPE and its nanocomposites contain different fillers. FTIR, Fourier transform infrared; HDPE, high-density polyethylene [Color figure can be viewed at wileyonlinelibrary.com]

\section{2 | Structural analysis}

Figure 1 presents the FTIR spectra of neat-HDPE and its PNCs. Pure HDPE shows two strong stretching and bending vibration at approximately 2912 and $2846 \mathrm{~cm}^{-1}$, representative of the $\mathrm{C}-\mathrm{H}$ symmetric and asymmetric stretching modes, respectively; furthermore, two $\mathrm{C}-\mathrm{H}$ bending vibration absorption bands at around 1462 and $718 \mathrm{~cm}^{-1}$. 33,34$]$ With filler incorporation, the chemical structure of polymer was well sustained as evidenced from the unaffected typical HDPE bands (Figure 1). This behavior indicates that the fillers were physical incorporated in the polymer constituents instead of developing chemical bonding throughout nanocomposites synthesis.

\section{3 | Thermal properties}

Table 2 and Figures 2 and 3 resume the thermal properties of neat PE and its nanocomposites. DSC measurements were performed in order to calculate any changes in the melting endotherm and crystallization temperature in the nanocomposites. It was observed that both $T_{\mathrm{m}}$ and $T_{\mathrm{c}}$ are slightly higher $\left(\sim 1^{\circ} \mathrm{C}\right)$ when compared to the neat $\mathrm{PE}$ falling in the error limit of the instrument. ${ }^{[35]}$ Unlike $T_{\mathrm{m}}$ and $T_{\mathrm{c}}$, an increase of $8 \%$ in the crystalline content was observed showing that the incorporation of magnetic filler favors the alignment and folding of polymer chains resulting in more orderly regions. The enhanced interfacial interaction among filler and matrix is considered the main reason to increase crystallinity. Many researchers have reported works on polymer filler interfaces, but the morphology and properties of the polymer at the interface are unclear understood. ${ }^{[36]}$ It can be concluded that the addition of filler does not show a significant effect on the melting and crystallizations temperature of the nanocomposites, although the formation of a higher degree of perfect crystal is the reason for the improvement in the percent crystallinity. ${ }^{[37]}$

T A B L E 2 Thermal properties of the neat PE and PE-C-M nanocomposites

\begin{tabular}{|c|c|c|c|c|c|c|c|c|}
\hline Samples & C-M $\mathbf{M}^{\mathrm{a}}(\%)$ & $M^{\mathbf{b}}(\%)$ & C-M $\mathbf{M}^{\mathbf{c}}(\%)$ & $T_{\mathrm{c}}\left({ }^{\circ} \mathrm{C}\right)$ & $T_{\mathrm{m}}\left({ }^{\circ} \mathbf{C}\right)$ & $X_{\mathrm{c}}(\%)$ & $T_{\text {onset }}\left({ }^{\circ} \mathbf{C}\right)$ & $T_{\max }\left({ }^{\circ} \mathbf{C}\right)$ \\
\hline PE-neat & 0 & 0 & 0 & 116 & 130 & 57 & 456 & 508 \\
\hline PE-C-neat & 2 & - & 1.2 & 117 & 130 & 62 & 465 & 513 \\
\hline $\mathrm{PE}-\mathrm{C}-\mathrm{Fe}$ & 2 & 0.9 & 1.6 & 117 & 130 & 62 & 465 & 513 \\
\hline PE-C-Co & 2 & 0.4 & 1.8 & 117 & 131 & 62 & 467 & 516 \\
\hline
\end{tabular}

Abbreviation: TGA, thermogravimetric analysis.

${ }^{\mathrm{a} A m o u n t}$ of filler (C-M) in the nanocomposite calculated from the yield.

${ }^{\mathrm{b}} \mathrm{M}=$ Amount of metal in the nanocomposite calculated from the TGA residue.

${ }^{\mathrm{c}}$ Amount of filler (C-M) in the nanocomposite calculated from the TGA residue. 


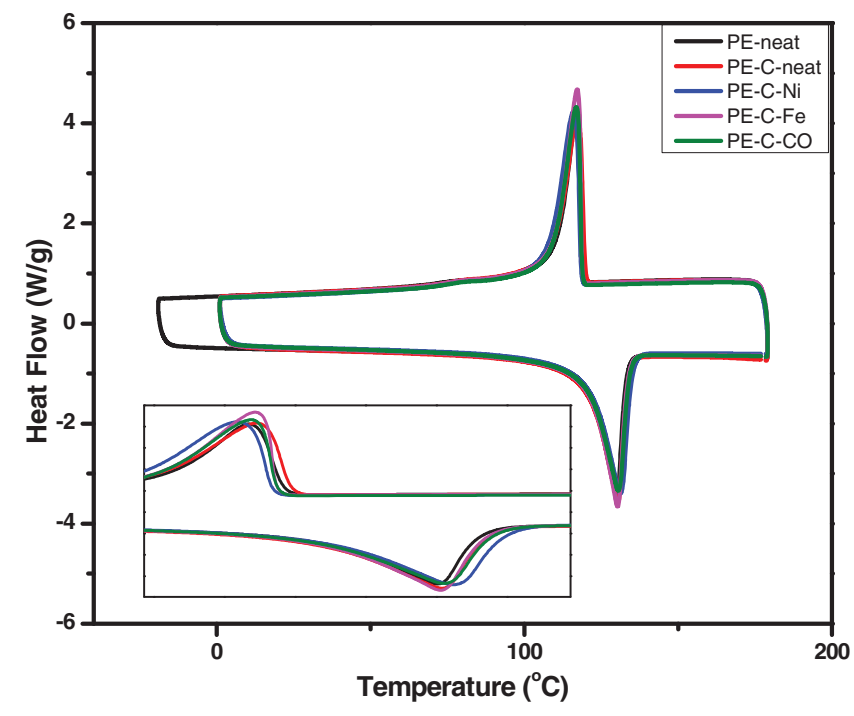

F I G URE 2 DSC graphs of the PE-neat and its nanocomposites. DSC, differential scanning calorimetry; PE, polyethylene [Color figure can be viewed at wileyonlinelibrary.com]

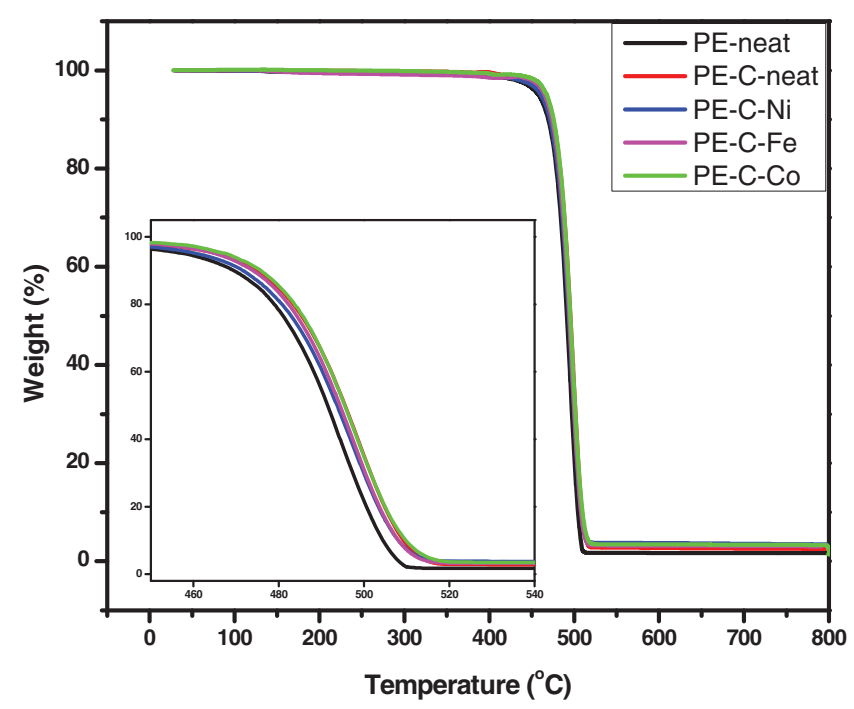

F I G U RE 3 TGA and DSC graphs of the PE-neat and its nanocomposites. DSC, differential scanning calorimetry; PE, polyethylene; TGA, thermogravimetric analysis [Color figure can be viewed at wileyonlinelibrary.com]

The study of thermal stability of polymeric materials is very important, being a limiting factor in both processing and applications. Thermal stability of neat HDPE and nanocomposites was measured by TGA under nitrogen atmosphere. The TGA evaluation demonstrated that with the addition of metal activated carbon the thermal stability of HDPE increases for all samples. The $T_{\text {onset }}$ degradation temperature of pristine $\mathrm{PE}\left(456^{\circ} \mathrm{C}\right)$ improved from $9^{\circ} \mathrm{C}$ to $11^{\circ} \mathrm{C}\left(465^{\circ} \mathrm{C}-467^{\circ} \mathrm{C}\right)$ with the incorporation of $2 \mathrm{wt} \%$ of metal activated carbon and the maximum degradation temperature of the nanocomposites increased from $508^{\circ} \mathrm{C}$ to $513^{\circ} \mathrm{C}-516^{\circ} \mathrm{C}$, showing an improvement for all nanocomposites samples (Figure 3).

The shifting of the degradation temperature to the higher value as seen in Table 2 and Figure 3 is in agreement with the rule of mixture as one of the constituents (C-M) is more thermally stable. Improvement in the degree of crystallinity and limitation of the molecular mobility around the metallic filler also play a role in this phenomenon, in accordance with percent crystallinity results. ${ }^{[38]}$

\section{4 | Morphological analysis}

Figure 4 presents SEM analysis of nanocomposites fractured surfaces. The unfilled PE and the nanocomposites show similar morphologies. On the other hand, nanocomposites showed more layered structure as compared to pristine $\mathrm{PE}$ with the polymer wrapped up around the fillers indicating strong interaction on the filler polymer interface. At this lower concentration of the filler, the fractured surface reveals the presence of primary filler particles in the form of platelets (Figure 4D), due to the inexpressive affinity to form agglomerates at this low filler loading. ${ }^{[39]}$

Figure 5 exhibits TEM images of the nanocomposites with 2 wt $\%$ of filler: (A) PE-C-neat-2\%, (B) PE-C-Fe-2\%, (B) $\mathrm{PE}-\mathrm{C}-\mathrm{Ni}-2 \%$, and (D) PE-C-Co-2\%, respectively. The micrographs show the presence of isolated particles and also some agglomerates, most prominent, in the case of sample PE-C-neat-2 wt\% and PE-C-Co- $2 \mathrm{wt} \%$. The presence of the black spots is attributed to the presence of metal particles in the polymer matrix; similar morphology and the presence of black spots are reported as metal particles by other researchers. ${ }^{[16,33]}$ In general, it was observed that the filler $(\mathrm{Fe}-\mathrm{C}$ and $\mathrm{Ni}-\mathrm{C})$ is rather uniformly dispersed in the $\mathrm{PE}$ matrix as compared to the neat-C and $\mathrm{Co}-\mathrm{C}$.

\section{5 | Mechanical properties}

Generally, the mechanical properties of composite materials depend on various factors, for instant the polymerfiller adhesion at interface, filler dispersion, aspect ratio of the filler, and the crystal structure of the polymer matrix. $^{[40]}$ Figure 6 shows the tensile testing of the dumbbell-shaped sample at an elongation rate of $50 \mathrm{~mm} \mathrm{~min}{ }^{-1}$. The initial region of tensile deformation is used to measure the tensile modulus and is an indication of the composite value of the constituent stiffness. The 
F I G U R E 4 SEM micrographs of: A, Neat-PE; B, PE-C-neat $2 \mathrm{wt} \%$; C, PE-C-Fe 2 wt $\%$; D, PE-C-Ni 2 wt $\%$; and E, PE-C-Co $2 \mathrm{wt} \%$ nanocomposites at a magnification of $5 \mu \mathrm{m}$. PE, polyethylene; SEM, scanning electron microscope
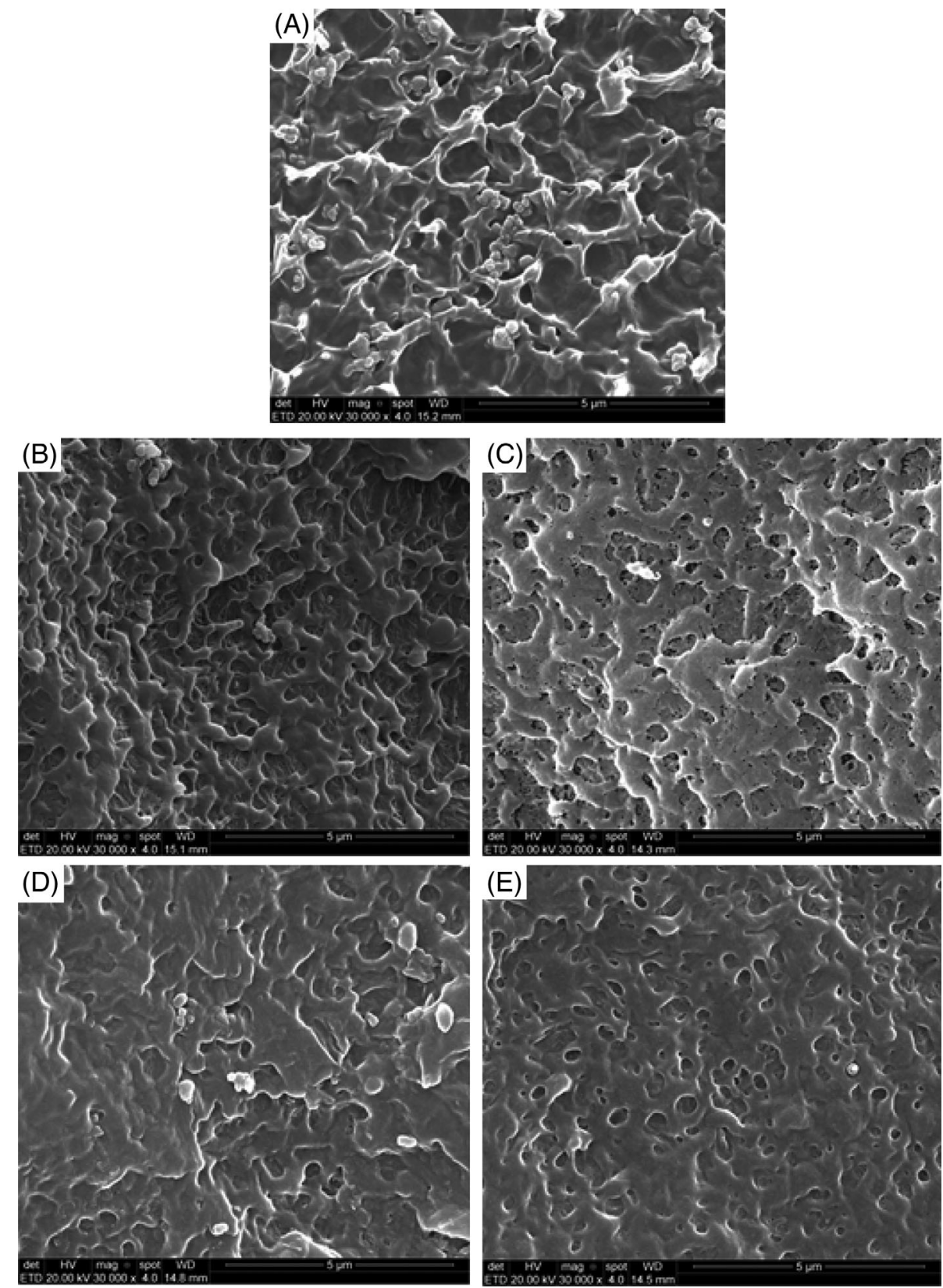

filler reinforced nanocomposites show the higher value of the tensile modulus compared to the unreinforced HDPE. The value of the modulus increases from $783 \mathrm{MPa}$ for HDPE to 956 and $1202 \mathrm{MPa}$ (22\% and 53\% enhancement) for PE-C-neat and PE-C-Ni samples, respectively. PE-CCo and PE-C-Fe samples show a slight decrease in the modulus. These impressive enhancements in the HDPE stiffness is endorsed to the identical distribution of the filler and to an improvement in the nanocomposite percent crystallinity as indicated from the DSC results. The highest improvement around 53\% in the modulus showed by the sample PE-C-Ni, exhibiting the highest increase of around $8 \%$ in the nanocomposite percent crystallinity as well. However, the decrease in the modulus in the case of sample PE-C-Fe is unclearly understood, but it can be due to the poor filler-matrix interface, acting as a barrier for effective load transfer from the matrix to the filler. ${ }^{[41]}$

As a general rule, with filler addition the elongation at break of PE matrix decreases. This is attributed to the filler strongly limiting the effort of the polymer chains, stopping them from elongating when they are beneath tension. ${ }^{[42]}$

\section{6 | Contact angle study}

For industrial applications of the polymer composite, the attraction toward water is a chief factor to be measured. The materials are superhydrophobic when the surface demonstrates high WCAs $\left(>150^{\circ}\right) .{ }^{[43]}$ The results of the 


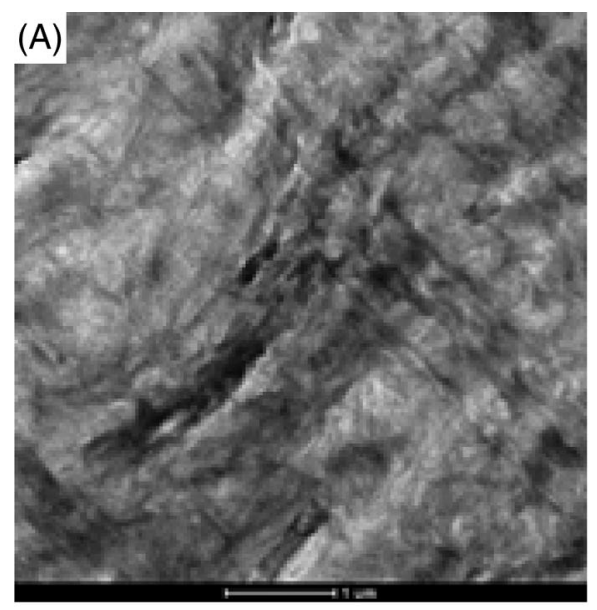

(B)

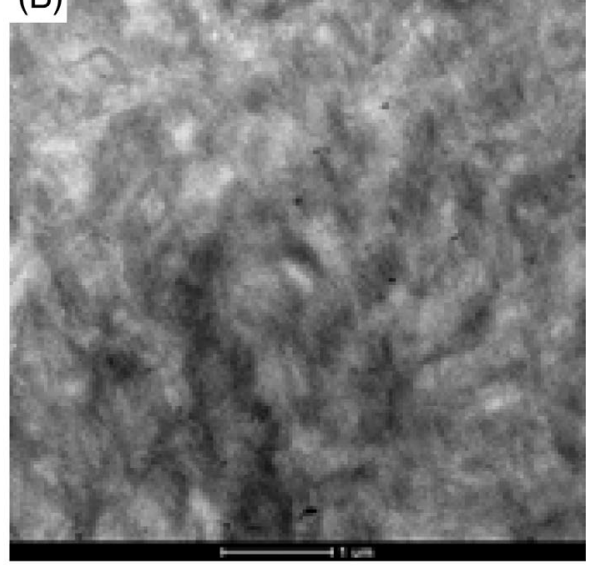

(C)

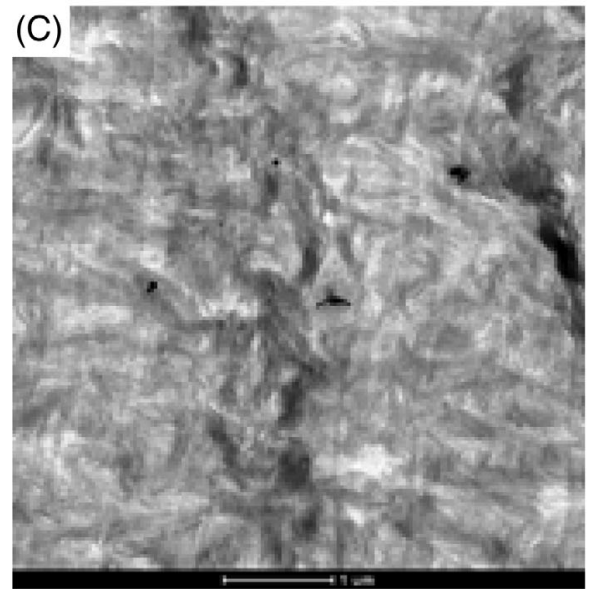

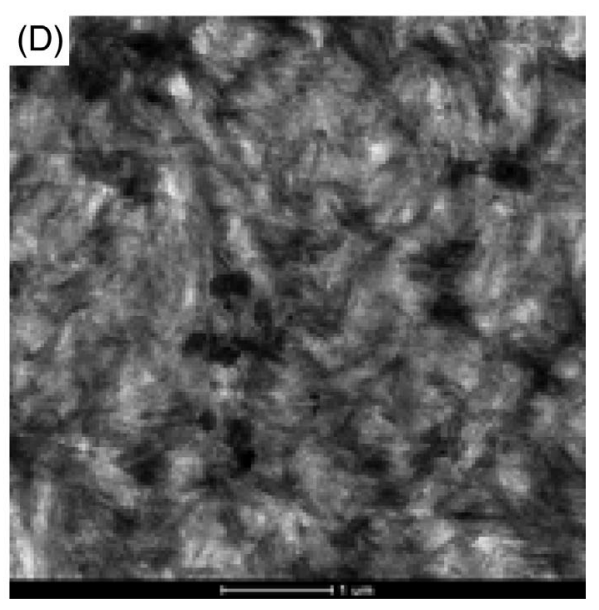

F I G U RE 5 TEM images of: A, PEC-neat 2 wt \%; B, PE-C-Fe 2 wt \%; C, PEC-Ni 2 wt \%; and D, PE-C-Co 2 wt $\%$ nanocomposites at a magnification of $100 \mathrm{~nm}$. PE, polyethylene; TEM, transmission electron microscopy
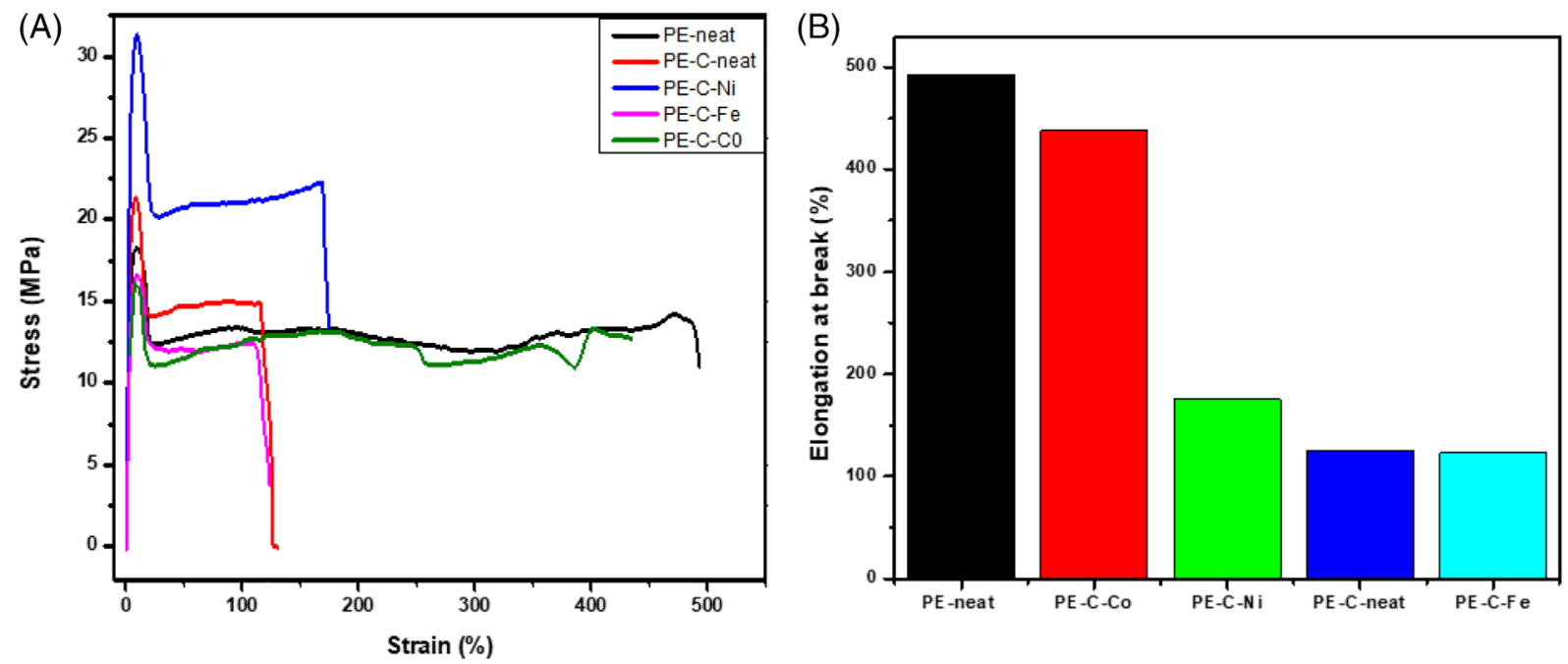

F I G U R E 6 A, Tensile stress-strain curve. B, Elongation at break point [Color figure can be viewed at wileyonlinelibrary.com]

water contact angle are listed in Table 3, showing that with filler addition, $\mathrm{C}-\mathrm{Co}$ and $\mathrm{C}-\mathrm{Fe}$, the contact angle is slightly increased demonstrating an improvement in the hydrophobic nature of the material. The rest of the two fillers (C-neat, C-Ni) demonstrates a similar contact angle as compared to the pristine PE.

\section{7 | Magnetic properties}

Figure 7 shows the magnetization $(M)$ vs $H$ loops, normalized to the respective saturation magnetizations $\left(M_{\mathrm{s}}\right)$, of the PE-C-Ni, PE-C-Fe, and PE-C-Co nanocomposites. The $H$ magnitude used here was sufficiently high to avoid 
minor-loop effects. ${ }^{[44,45]}$ The values of the remnant magnetization $\left(M_{\mathrm{r}}\right)$, normalized to $M_{\mathrm{s}}$, and of the coercive field $\left(H_{c}\right)$ of these samples are given in Table 3. Although the PE$\mathrm{C}$-Ni nanocomposites saturate in magnetic fields lower than those with $\mathrm{Fe}$ (see the insets in Figure 7) and also have lower $H_{\mathrm{c}}$ values, their $M_{\mathrm{r}} / M_{\mathrm{s}}$ value is twice greater. The PEC-Co samples have small coercivity as well but their $M$ keeps increasing even in the highest-field region (ie, superparamagnetic-type behavior, indicating welldispersed Co entities with very small sizes), so it is impossible to estimate $M_{\mathrm{r}} / M_{\mathrm{s}}$ for these nanocomposites.

Wilson et al ${ }^{[46]}$ synthesized poly(methyl methacrylate) (PMMA) iron nanoparticles embedded nanocomposites presenting a coercivity of 260 Oe and $M_{\mathrm{r}} / M_{\mathrm{S}}=0.15$ with the addition of $10 \mathrm{wt} \%$ of iron nanoparticles in the PMMA matrix. Yurkov et $\mathrm{al}^{[47]}$ introduced cobalt nanoparticles into polytetrafluoroethylene matrix, the sample with the highest concentration $3.9 \mathrm{wt} \%$ of Co showing a coercivity of $0.3 \mathrm{kOe}$ and magnetization of $0.4 \mathrm{emu} \mathrm{g}^{-1}$ at $H=6 \mathrm{kOe}$ at room temperature. In contrast, our samples show coercivity of $175 \mathrm{Oe}$ and $M_{\mathrm{r}} / M_{\mathrm{s}}=0.14$ with a very small amount of metal nanoparticles $0.9 \mathrm{wt} \%$ at a low applied field value of $2 \mathrm{kOe}$. This outstanding magnetic response

T A B L E 3 Contact angle and magnetic properties of the nanocomposites

\begin{tabular}{lllll} 
Samples & $\mathbf{C - M}^{\mathbf{a}}(\boldsymbol{\%})$ & $\boldsymbol{H}_{\mathbf{c}}(\mathbf{O e})$ & $\boldsymbol{M}_{\mathbf{r}} / \boldsymbol{M}_{\mathbf{s}}$ & WCA $\left({ }^{\circ}\right)$ \\
\hline PE-neat & 0 & - & - & $73.9 \pm 0.5$ \\
\hline PE-C-neat & - & - & - & $72.0 \pm 0.9$ \\
\hline PE-C-Ni & 0.5 & $100 \pm 5$ & $0.14 \pm 0.01$ & $70.2 \pm 0.2$ \\
\hline PE-C-Fe & 0.9 & $175 \pm 5$ & $0.07 \pm 0.01$ & $77.3 \pm 0.6$ \\
\hline PE-C-Co & 0.4 & $120 \pm 5$ & - & $76.0 \pm 1.7$ \\
\hline
\end{tabular}

Abbreviations: PE, polyethylene; TGA, thermogravimetric analysis; WCA, water contact angle.

${ }^{\mathrm{a}} \mathrm{M}=$ Amount of metal in the nanocomposite calculated from the TGA residue. at such low concentration of the magnetic filler corresponds to the immobilization of the magnetic entities in the nonmagnetic carbon part preventing the magnetic induced agglomeration and facilitating the uniform filler dispersion in the PE matrix.

In order to estimate the effects of magnetic interactions in each of the nanocomposites, we employed the recently introduced $\delta M_{\mathrm{R}}$ plot technique. ${ }^{[48]}$ Nonzero deviations of $\delta M_{\mathrm{R}}(H)$ in systems with uniaxial anisotropy are ascribed to magnetic coupling. The negative interactions (dipolar-like, stabilizing the demagnetized state) that our magnetic samples present are rather weak, indicating a very good dispersion of the magnetic entities in the polymer matrix, preventing particle aggregation.

The nanocomposite with the greatest coercivity, the $\mathrm{PE}-\mathrm{C}-\mathrm{Fe}$ one, shows the most pronounced, visibly negative $\delta M_{\mathrm{R}}$ curve. This behavior could be attributed to the highest $M_{\mathrm{S}}$ value of $\mathrm{Fe}$ as compared to those of $\mathrm{Ni}$ and $\mathrm{Co}$, and, most importantly, to the fact that the metal $(\mathrm{Fe})$ amount in these nanocomposites is higher by almost a factor of two when compared to $\mathrm{Ni}$ or Co samples as seeing in Table 3. This higher concentration of magnetic grains could also be the cause for the low $M_{\mathrm{r}} / M_{\mathrm{s}}$ value of the nanocomposites that contain $\mathrm{Fe}$, having in mind that dipolar coupling results in closed magnetic flux configurations leading to a decrease of $M_{\mathrm{r}} / M_{s}$.

\section{5 | CONCLUSIONS}

The metal activated carbon nanocomposites from the biomass (a cost-effective source) were synthesized and used as fillers in PE nanocomposites by melt mixing technique. The activation of the carbon by metals, such as $\mathrm{Co}, \mathrm{Ni}$, and $\mathrm{Fe}$, was carried out to encapsulate them by amorphous carbon, thus controlling the oxidation and increasing the interaction with the apolar PE matrix. The nanocomposites FTIR spectra show PE characteristic
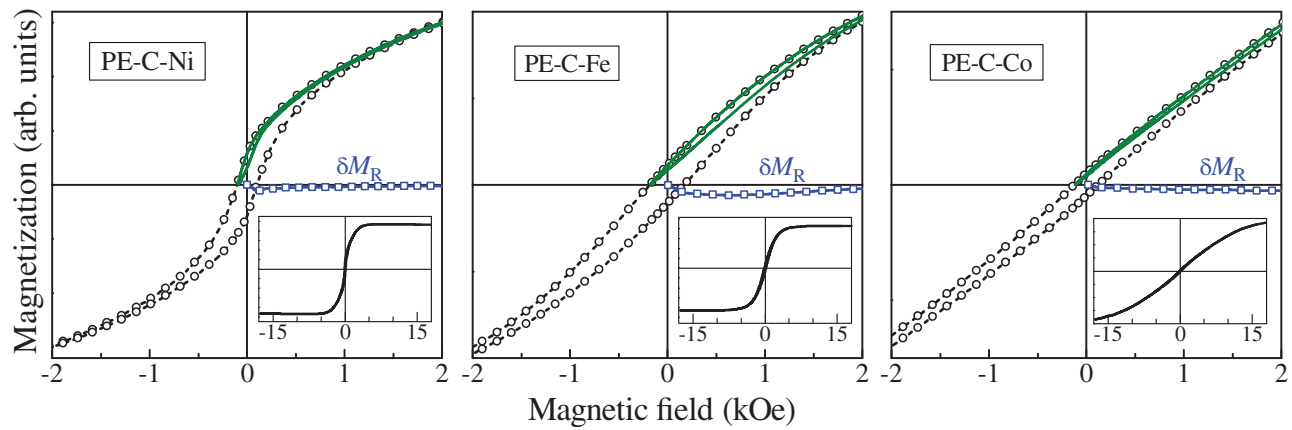

FI G U RE 7 Low-field region of the magnetization major hysteresis loops (circles), recoil curves (solid lines) with recoil field equal to $H_{\mathrm{c}}$ and the respective $\delta M_{\mathrm{R}}$ interaction plots (rectangles) of the PE-Ni-C, PE-Fe-C, and PE-Co-C nanocomposites. The insets show the major loops in the whole magnetic field region. PE, polyethylene [Color figure can be viewed at wileyonlinelibrary.com] 
peaks presenting no evidence of peak shifting with filler addition. This behavior is in agreement with the physical interaction of the polymer filler interface without any chemical bonding. The nanocomposite thermal stability was improved with the filler addition, presenting enhancements of $11^{\circ} \mathrm{C}$ and $8^{\circ} \mathrm{C}$, in the onset degradation and maximum degradation temperatures, respectively. The percent crystallinity was improved by $8 \%$ indicating the formation of more perfect crystals. An improvement of up to approximately 53\% was observed in the elasticity module. The improvement in the mechanical and thermal properties, along with the attainment of the magnetic properties at room temperature in the polymer, spreads its applications for engineering of diverse procedures. These consequences are important because most works reported in literature in this area display magnetic finding only at below ambient temperatures. A higher coercivity was observed for PE-C-Fe nanocomposite, and is noted that the amount of the magnetic entities of $\mathrm{Fe}$ is higher in the activated carbon as compared to the Co and $\mathrm{Ni}$ activated carbon. The weaker demagnetizing interactions of the magnetic particles indicate a uniform dispersion of the filler. These results are supported by the TEM and SEM images where uniform dispersion of the weakly interacting magnetic filler is also observed. Moreover, magnetism is also demonstrated by the statistics that nanocomposite materials are intensely attracted by a magnet. These results are remarkable since the application of external magnetic field allows the separation of the material from the medium.

\section{ACKNOWLEDGMENTS}

The research has been partly financed by the Brazilian agencies Conselho Nacional de Desenvolvimento Científico e Tecnológico (CNPq) under grants 305796/2016-0, 314429/2018-2, and 422740/2018-7 and Coordenação de Aperfeiçoamento de Pessoal de Nível Superior (CAPES)-Finance Code 001.

\section{CONFLICT OF INTEREST}

The authors declare no conflicts of interest.

\section{ORCID}

Sandra Einloft (1) https://orcid.org/0000-0003-1364-0325

\section{REFERENCES}

[1] X. Chen, S. Wei, C. Gunesoglu, J. Zhu, C. S. Southworth, L. Sun, A. B. Karki, D. P. Young, Z. Guo, Macromol. Chem. Phys. 2010, 211(16), 1775. https://doi.org/10.1002/macp. 201000153.

[2] K. Holmberg, Curr. Opin. Colloid. Interface Sci. 2003, 8, 145. https://doi.org/10.1016/S1359-0294.
[3] K. W. Putz, O. C. Compton, M. J. Palmeri, S. B. T. Nguyen, L. C. Brinson, Adv. Funct. Mater. 2010, 20(19), 3322. https:// doi.org/10.1002/adfm.201000723.

[4] M. Nisar, C. Bergmann, J. Geshev, R. Quijada, G. B. Galland, Polymer 2016, 97, 131. https://doi.org/10.1016/j.polymer.2016. 05.029 .

[5] M. Nisar, M. D. G. Sebag Bernd, L. C. P. da Silva Filho, J. Geshev, N. R. de Souza Basso, G. G. Barrera, J. Appl. Polym. Sci. 2018, 135, 46820. https://doi.org/10.1002/app.46820.

[6] R. Haggenmueller, C. Guthy, J. R. Lukes, J. E. Fischer, K. I. Winey, Macromolecules 2007, 40(7), 2417. https://doi.org/ 10.1021/ma0615046.

[7] W. Bauhofer, J. Z. Kovacs, Compos. Sci. Technol. 2009, 69(10), 1486. https://doi.org/10.1016/j.compscitech.2008.06.018.

[8] T. Kashiwagi, F. Du, J. F. Douglas, K. I. Winey, R. H. Harris, J. R. Shields, Nat. Mater. 2005, 4(12), 928. https://doi.org/10. 1038/nmat1502.

[9] J. Zhu, S. Wei, X. Chen, A. B. Karki, D. Rutman, D. P. Young, Z. Guo, J. Phys. Chem. C 2010, 114(19), 8844. https://doi.org/ 10.1021/jp1020033.

[10] Z. Guo, S. Park, H. T. Hahn, S. Wei, M. Moldovan, A. B. Karki, D. P. Young, J. Appl. Phys. 2007, 101(9), 09M511-1. https://doi. org/10.1063/1.2711074.

[11] Z. Guo, S. E. Lee, H. Kim, S. Park, H. T. Hahn, A. B. Karki, D. P. Young, Acta Mater. 2009, 57(1), 267. https://doi.org/10. 1016/j.actamat.2008.09.024.

[12] J. Zhu, S. Wei, Y. Li, L. Sun, N. Haldolaarachchige, D. P. Young, C. Southworth, A. Khasanov, Z. Luo, Z. Guo, Macromolecules 2011, 44(11), 4382. https://doi.org/10.1021/ ma102684f.

[13] J. Zhu, S. Wei, J. Ryu, L. Sun, Z. Luo, Z. Guo, ACS Appl. Mater. Interfaces 2010, 2(7), 2100. https://doi.org/10.1021/ am100361h.

[14] P. Kim, N. M. Doss, J. P. Tillotson, P. J. Hotchkiss, M. J. Pan, S. R. Marder, J. Li, J. P. Calame, J. W. Perry, ACS Nano. 2009, 3(9), 2581.

[15] J. Zhu, S. Wei, J. Ryu, M. Budhathoki, G. Liang, Z. Guo, J. Mater. Chem. 2010, 20(23), 4937. https://doi.org/10.1039/ c0jm00063a.

[16] M. Nisar, P. S. Thue, C. A. Heck, J. L. Salazar Cuaila, J. Geshev, E. C. Lima, M. M. Jacobi, G. B. Galland, Eur. Polym. J. 2018, 99, 378. https://doi.org/10.1016/j.eurpolymj. 2017.12.044.

[17] M. el Achaby, A. Qaiss, Mater. Des. 2013, 44, 6151. https://doi. org/10.1021/la960929q.

[18] D. Zhang, S. Wei, C. Kaila, X. Su, J. Wu, A. B. Karki, D. P. Young, Z. Guo, Nanoscale 2010, 2(6), 917. https://doi. org/10.1039/c0nr00065e.

[19] S. Kalia, S. Kango, A. Kumar, Y. Haldorai, B. Kumari, R. Kumar, Colloid Polym. Sci. 2014, 292(9), 2025. https://doi. org/10.1007/s00396-014-3357-y.

[20] Y. Lu, Y. Yin, B. T. Mayers, Y. Xia, Nano Lett. 2002, 2(3), 183. https://doi.org/10.1021/nl015681q.

[21] C. Boyer, V. Bulmus, P. Priyanto, W. Y. Teoh, R. Amal, T. P. Davis, J. Mater. Chem. 2009, 19(1), 111. https://doi.org/ 10.1039/b815202k.

[22] J. E. An, G. W. Jeon, Y. G. Jeong, Fibers Polym. 2012, 13(4), 507. https://doi.org/10.1007/s12221-012-0507-z. 
[23] R. Méndez, B. Constant, C. Garzon, M. Nisar, S. M. B. Nachtigall, R. Quijada, Polymer 2017, 130, 10. https:// doi.org/10.1016/j.polymer.2017.09.063.

[24] M. El Achaby, A. Qaiss, Mater. Des. 2013, 44, 81. https://doi. org/10.1016/j.matdes.2012.07.065.

[25] M. A. Milani, R. Quijada, N. R. S. Basso, A. P. Graebin, G. B. Galland, J. Polym. Sci. Part A: Polym. Chem. 2012, 50(17), 3598. https://doi.org/10.1002/pola.26149.

[26] A. A. Koval'chuk, A. N. Shchegolikhin, V. G. Shevchenko, P. M. Nedorezova, A. N. Klyamkina, A. M. Aladyshev, Macromolecules 2008, 41(9), 3149. https://doi.org/10.1021/ ma800297e.

[27] S. P. Bao, S. C. Tjong, Mater. Sci. Eng. A 2008, 485(1-2), 508. https://doi.org/10.1016/j.msea.2007.08.050.

[28] M. A. Prado, G. Dias, C. Carone, R. Ligabue, A. Dumas, C. le Roux, P. Micoud, F. Martin, S. Einloft, J. Appl. Polym. Sci. 2015, 132(16), 1. https://doi.org/10.1002/app.41854.

[29] G. Dias, M. Prado, R. Ligabue, M. Poirier, C. le Roux, F. Martin, S. Fery-Forgues, S. Einloft, Appl. Clay Sci. 2018, 158, 37. https://doi.org/10.1016/j.clay.2018.03.012.

[30] G. Dias, M. Prado, C. Carone, R. Ligabue, A. Dumas, C. le Roux, P. Micoud, F. Martin, S. Einloft, Macromol. Symp. 2016, 367(1), 136. https://doi.org/10.1002/masy.201500141.

[31] P. S. Thue, E. C. Lima, J. M. Sieliechi, C. Saucier, S. L. P. Dias, J. C. P. Vaghetti, F. S. Rodembusch, F. A. Pavan, J. Colloid Interface Sci. 2017, 486, 163. https://doi.org/10.1016/j.jcis.2016. 09.070.

[32] J. M. Sieliechi, P. S. Thue, Desalin. Water Treat. 2015, 55(4), 986. https://doi.org/10.1080/19443994.2014.922504.

[33] Q. He, T. Yuan, J. Zhu, Z. Luo, N. Haldolaarachchige, L. Sun, A. Khasanov, Y. Li, D. P. Young, S. Wei, Z. Guo, Polymer 2012, 53(16), 3642. https://doi.org/10.1016/j.polymer.2012.06.010.

[34] M. S. Mehmood, A. Sanawar, N. Siddiqui, T. Yasin, Polym. Bull. 2017, 74(1), 213. https://doi.org/10.1007/s00289-0161709-0.

[35] T. Kuila, S. Bose, A. K. Mishra, P. Khanra, N. H. Kim, J. H. Lee, Polym. Test. 2012, 31(1), 31. https://doi.org/10.1016/ j.polymertesting.2011.09.007.

[36] F. V. Ferreira, W. Franceschi, B. R. C. Menezes, F. S. Brito, K. Lozano, A. R. Coutinho, L. S. Cividanes, G. P. Thim, Appl. Surf. Sci. 2017, 410, 267. https://doi.org/10.1016/j.apsusc.2017.03.098.
[37] M. A. L. Manchado, L. Valentini, J. Biagiotti, J. M. Kenny, Carbon 2005, 43(7), 1499. https://doi.org/10.1016/j.carbon.2005.01.031.

[38] A. Chatterjee, B. L. Deopura, J. Appl. Polym. Sci. 2006, 100(5), 3574. https://doi.org/10.1002/app.22864.

[39] F. R. Costa, B. K. Satapathy, U. Wagenknecht, R. Weidisch, G. Heinrich, Eur. Polym. J. 2006, 42(9), 2140. https://doi.org/ 10.1016/j.eurpolymj.2006.04.005.

[40] C. Zhao, H. Qin, F. Gong, M. Feng, S. Zhang, M. Yang, Polym. Degrad. Stab. 2005, 87(1), 183. https://doi.org/10.1016/j. polymdegradstab.2004.08.005.

[41] W. Zheng, X. Lu, S. C. Wong, J. Appl. Polym. Sci. 2004, 91(5), 2781. https://doi.org/10.1002/app.13460.

[42] M. Nisar, M. G. Sebag Bernd, L. C. Pinto da Silva Filho, J. Geshev, G. Galland, Polym. Adv. Technol. 2019, 30(2), 457. https://doi.org/10.1002/pat.4493.

[43] G. R. Chagas, D. E. Weibel, Polym. Bull. 2017, 74(6), 1965. https://doi.org/10.1007/s00289-016-1817-x.

[44] A. Harres, M. Mikhov, V. Skumryev, A. M. H. de Andrade, J. E. Schmidt, J. Geshev, J. Magn. Magn. Mater. 2016, 402, 76. https://doi.org/10.1016/j.jmmm.2015.11.046.

[45] J. Geshev, J. Appl. Phys. 2009, 104, 066108. https://doi.org/10. 1063/1.3098262.

[46] J. L. Wilson, P. Poddar, N. A. Frey, H. Srikanth, K. Mohomed, J. P. Harmon, S. Kotha, J. Wachsmuth, J. Appl. Phys. 2004, 95 (3), 1439. https://doi.org/10.1063/1.1637705.

[47] G. Y. Yurkov, A. S. Fionov, Y. A. Koksharov, V. V. Koleso, S. P. Gubin, Inorg. Mater. 2007, 43(8), 834. https://doi.org/10. 1134/s0020168507080055.

[48] J. Geshev, J. Magn. Magn. Mater. 2018, 467, 135. https://doi. org/10.1016/j.jmmm.2018.07.009.

How to cite this article: Nisar M, Thue PS, Maghous MB, Geshev J, Lima EC, Einloft S. Metal activated carbon as an efficient filler for highdensity polyethylene nanocomposites. Polymer Composites. 2020;41:3184-3193. https://doi.org/10. $1002 /$ pc. 25610 